\title{
Avaliação radiográfica dos efeitos do aparelho Distal Jet nas distalizações intra-bucais: Um estudo piloto*
}

\author{
Giordani Santos SILVEIRA**, Luiz Fernando ETO***
}

\section{Resumo}

Freqüentemente, o tratamento da má oclusão de Classe II requer a distalização de molares superiores. $\mathrm{O}$ aparelho extrabucal foi o primeiro sistema criado para este fim, e ao longo de mais de um século de uso, provou sua eficiência. Entretanto, apresenta como grande desvantagem, o impacto estético negativo, já que os seus resultados estão atrelados à sistemática colaboração dos pacientes. Assim, vários aparelhos distalizadores intra-bucais foram desenvolvidos na expectativa de eliminar a necessidade de colaboração do paciente, dentre eles, se destaca o Distal Jet. Segundo Carano e Testa (1996), ele é o único mecanismo intrabucal capaz de distalizar os molares de corpo. Para avaliar os efeitos deste aparelho, um estudo piloto foi desenvolvido, utilizando-se seis adolescentes (idade média: 13,08 anos), portadores de má oclusão de Classe II dentária e padrão facial semelhante entre si. Telerradiografias laterais pré- e pós-distalização foram realizadas. O tempo médio desta fase foi de 4,5 meses. Mudanças de tecidos moles, esqueléticas e dentárias foram determinadas, comparando-se as medidas dos cefalogramas iniciais e finais. Utilizando-se o teste de Wilcoxon, as variáveis que apresentaram diferença significativa foram: inclinação distal da coroa do segundo molar, distalização do primeiro molar e distalização do segundo molar. O Distal Jet mostrou ser eficiente na distalização dos molares superiores, sobretudo pelo pequeno componente de inclinação dos primeiros molares. A perda de ancoragem e o aumento da altura facial ântero-inferior não apresentaram significância estatística.

Palavras-chave: Classe II. Aparelhos distalizadores intra-bucais. Distal Jet.

\section{INTRODUÇÃO}

À luz do avanço do conhecimento contemporâneo, sabe-se que a má oclusão de Classe II não se constitui como uma simples síndrome, mas como um conjunto de vários subtipos. Ela pode ser resultante, tanto de uma discrepância dentária, quanto de um desequilíbrio entre as bases ósseas: protrusão maxilar, retrusão mandibular ou a combinação de ambas, 23,33 . Considerando as modalidades de tratamento das más oclusões de Classe II dentárias, a distalização de molares superiores provou ser desejável e factível ${ }^{4,8,14,15,37}$.

* Resumo da Monografia de Especialização em Ortodontia e Ortopedia Facial (Universidade de Itaúna - MG).

** Especialista em Ortodontia e Ortopedia Facial pela Universidade de Itaúna - MG.

*** Mestre em Ortodontia pela PUC-MG. Professor do Curso de Especialização em Ortodontia e Ortopedia Facial da Universidade de Itaúna - MG. 
Na literatura são relatados vários tipos de aparelhos e sistemas que se propõem a distalizar os molares superiores. O primeiro deles, é o aparelho extrabucal introduzido por Kingsley, citado por Gould ${ }^{19}$, no final do século XIX, ainda largamente utilizado e com expressivo respaldo científico ${ }^{2,20,26,28,29,30,36}$. No entanto, por ser um aparelho removível que apresenta componentes localizados fora da cavidade bucal, muitos pacientes manifestam resistência em utilizá-lo, prejudicando sobremaneira, o resultado planejado pelo ortodontista $714,31,34,40$. Além disso, existem relatos de lesões oculares e faciais invasivas relacionadas à utilização deste aparelho $0^{1,11,24,38}$.

Neste contexto, constata-se uma grande demanda por mecanismos distalizadores, que não dependam da cooperação do paciente. Tulloch, Phillips e Proffit ${ }^{41}$ afirmaram que só entre os anos de 1980 a 1990, cerca de 130 artigos, apresentando 14 sistemas diferentes de aparelhos ou modalidades de tratamento para a correção da má oclusão de Classe II, foram descritos nos quatro maiores periódicos de língua inglesa. Os mecanismos mais difundidos e, conseqüentemente mais estudados, foram: os magnetos repelentes ${ }^{4,17,18,25}$, os fios superelásticos $\mathrm{NiTi}^{15,32}$, as molas abertas de $\mathrm{NiTi}{ }^{12,15,16}$, o Jones Jig ${ }^{27,37,40}$ e o Pendulum ${ }^{8,14,22}$. Mesmo exigindo uma cooperação mínima do paciente, muitos desses aparelhos distalizadores intra-bucais, ainda, produziam efeitos adversos, tais como: inclinação acentuada da coroa do molar superior e perda de ancoragem, de moderada a severa.

Carano e Testa $^{9}$ desenvolveram o Distal Jet, um aparelho distalizador intra-bucal capaz de distalizar os molares de corpo, sem necessitar da cooperação do paciente. Além disso, ele poderia ser, facilmente, convertido num aparelho de Nance passivo nos molares, para manter a distalização obtida. Baseados no estudo de dois casos clínicos, os autores afirmaram que os índices de movimentação dos molares superiores foi comparável aos reportados com o uso dos magnetos repelentes e do Jones Jig, mas que eles foram obtidos, sem inclinação ou rotação.
Com o intuito de aumentar a versatilidade do aparelho Distal Jet, Bowman ${ }^{7}$ introduziu modificações ao desenho original do aparelho proposto por Carano e Testa ${ }^{9}$. A primeira delas, foi a introdução de um anel de ativação com dois parafusos, o que facilitou a conversão do aparelho ativo, em um aparelho passivo de Nance. A outra, foi a sugestão de se inserir um helicóide no fio baioneta para aumentar o comprimento de fio e possibilitar a correção de rotação molar superior com forças mais apropriadas.

Ngantung, Nanda e Bowman ${ }^{35}$ realizaram um estudo, para analisar os efeitos do tratamento com o uso do aparelho Distal Jet, associado ao aparelho fixo pré-ajustado superior. Para isso, utilizaram radiografias cefalométricas, tiradas antes e após a distalização dos molares superiores, em 33 adolescentes. Os resultados mostraram que o aparelho é, realmente, capaz de distalizar os molares superiores com pouco índice de inclinação distal dos mesmos. Entretanto, ocorreu uma grande taxa de perda de ancoragem, na forma de mesialização dos pré-molares de ancoragem e inclinação labial dos incisivos. Não ocorreu aumento da altura facial inferior.

Devido à escassez de literatura disponível, no que tange à avaliação da eficácia deste aparelho, é que decidiu-se pela realização do presente trabalho. Um estudo piloto foi desenvolvido, com os objetivos de propor uma amostragem e uma metodologia sistematicamente padronizadas, e de determinar os efeitos causados pelo uso do aparelho Distal Jet, tanto nos molares, pré-molares de ancoragem e incisivos superiores, quanto no posicionamento espacial da mandíbula e perfil mole.

\section{MATERIAIS E MÉTODOS Materiais Amostragem}

A fim de eliminar as deficiências na padronização no campo da amostra, encontradas em vários trabalhos prévios, este estudo prospectivo adotou alguns critérios para a inclusão dos indivíduos na amostra, dentre os quais se destacam: 
1) relação molar de Classe II bilateral;

2) má oclusão de Classe I esquelética ou Classe II esquelética suave, de acordo com os valores demonstrados por Silva Filho ${ }^{39}$;

3) padrão esquelético vertical normal, segundo os valores relatados por Silva Filho ${ }^{39}$ e;

4) nenhuma outra modalidade de tratamento utilizada como: aparelhagem fixa, aparelho extra-bucal ou elásticos, durante o período de observação.

Foram selecionados para a pesquisa seis pacientes (três do gênero masculino e três do gênero feminino), leucodermas brasileiros, com idades compreendidas entre 12.1 a 14.4 anos. A ausência do segundo molar superior na cavidade bucal não foi levada em conta, de modo que dois pacientes apresentavam os segundos molares irrompidos bilateralmente e um paciente tinha, apenas, um segundo molar presente na cavidade bucal.

\section{Aparelho Distal Jet}

O Distal Jet é um aparelho distalizador intrabucal fixo, que utiliza ancoragem dentomucosuportada caracterizada por um aparelho de Nance modificado que pode estar unido tanto aos primeiros quanto aos segundos pré-molares superiores. O seu componente ativo, localizado por lingual, é composto de um mecanismo de tubo telescópico associado aos anéis de ativação e a molas abertas

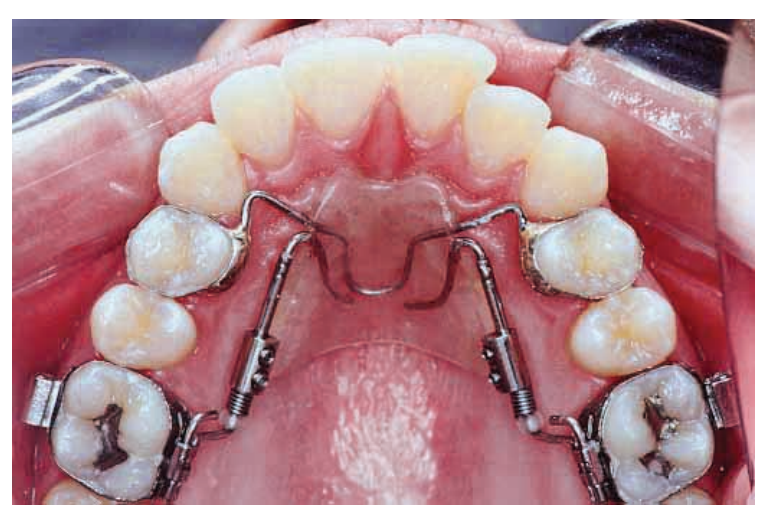

FIGURA 1 - Aspecto oclusal do aparelho Distal Jet. 0 mecanismo de tubo telescópico está localizado de modo que as molas comprimidas de NiTi exerçam uma força distal próxima aos centros de resistência dos primeiros molares superiores com o objetivo de promover movimentação de corpo. de níquel-titânio (Fig. 1). Estas molas são précalibradas em 180 gramas e 240 gramas quando totalmente comprimidas, sendo, respectivamente, indicadas na ausência e na presença dos segundos molares superiores.

Todos os pacientes deste estudo utilizaram o aparelho Distal Jet do "Kit" da American Orthodontics $^{\circledR}$. Foram utilizados como dentes de ancoragem os primeiros pré-molares superiores, em função de haver dois indivíduos da amostra que não apresentavam os segundos pré-molares superiores irrompidos. As molas abertas de níquel-titânio de 240 gramas foram usadas, bilateralmente, em todos os pacientes, devido ao fato de três pacientes da amostra apresentarem pelo menos, um segundo molar superior irrompido.

\section{Métodos}

O aparelho Distal Jet foi fixado como um corpo único e, posteriormente à sua cimentação, esse aparelho foi ativado, bilateralmente, através da compressão total das molas abertas de NiTi pelos anéis de ativação, e aperto do parafuso mesial destes últimos com a chave sextavada. Para manter os níveis de força, o aparelho foi reativado da mesma maneira em intervalos de quatro semanas.

A conversão do aparelho ativo em um aparelho passivo de Nance (Fig. 2) só foi procedida, quando as cúspides mésio-vestibulares dos primeiros mo-

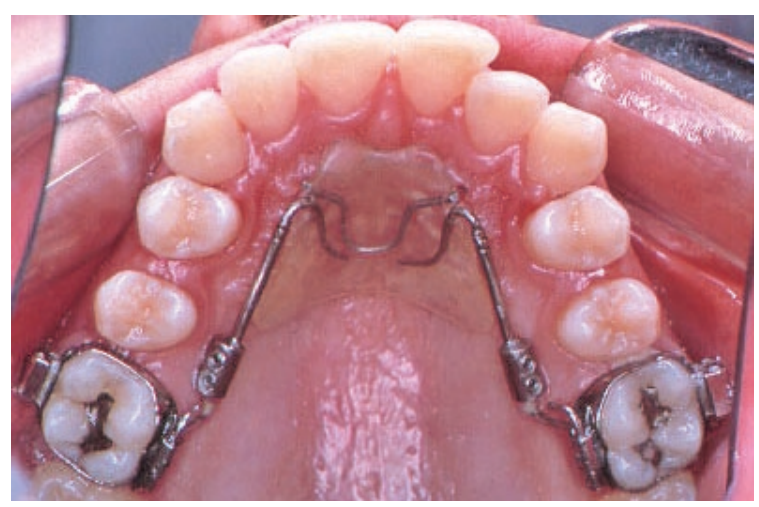

FIGURA 2 - Aspecto oclusal do aparelho de Nance passivo obtido a partir do Distal Jet após remoção dos anéis dos primeiros pré-molares. 


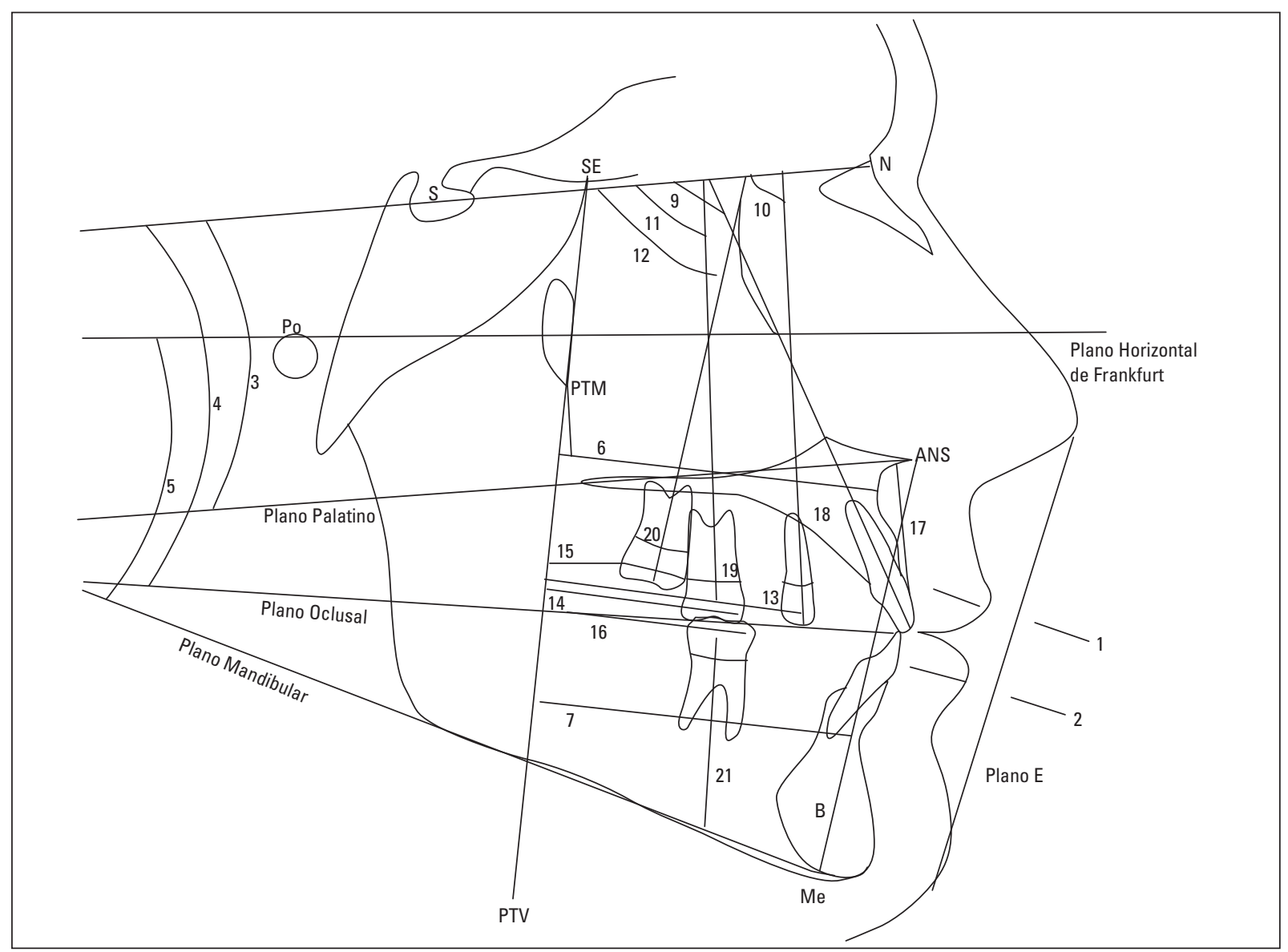

FIGURA 3 - Planos e medidas cefalométricas (de tecidos moles, esqueléticas e dentárias) usados no estudo: 1) Lábio superior - Plano E; 2) Lábio inferior - Plano E; 3) Ângulo SN - Plano palatino; 4) Ângulo SN - Plano oclusal; 5) Ângulo Frankfurt - Plano mandibular; 6) PTV - Ponto A; 7) PTV - Ponto B; 8) ANS - Me; 9) SN - Incisivo superior; 10) SN - Primeiro pré-molar superior; 11) SN - Primeiro molar superior; 12) SN - Segundo molar superior; 13) PTV - Centróide primeiro pré-molar superior; 14) PTV - Centróide primeiro molar superior; 15) PTV - Centróide segundo molar superior; 16) PTV - Centróide primeiro molar inferior; 17) PP - Borda incisal do incisivo superior; 18) PP - Centróide primeiro pré-molar superior; 19) PP - Centróide primeiro molar superior; 20) PP - Centróide segundo molar superior e 21) MP - Centróide primeiro molar inferior.

FONTE: NGANTUNG, NANDA e BOWMAN ${ }^{35}$, 2001. p.180

lares superiores estavam $1 \mathrm{~mm}$ posteriores aos sulcos vestibulares dos primeiros molares inferiores.

As telerradiografias laterais de crânio, feitas, previamente, ao início do tratamento ortodôntico (primeira fase) e, imediatamente após a conversão do aparelho Distal Jet num aparelho de Nance passivo, foram feitas em todos os pacientes do estudo, sendo tiradas pelo mesmo operador técnico, em um mesmo centro de documentação ortodôntica. O intervalo de tempo entre a interrupção da fase ativa de distalização (conversão do aparelho ativo para um aparelho passivo de Nance) e a radiografia cefalométrica final, foi inferior a 24 horas.
A análise cefalométrica utilizada no estudo baseou-se na empregada no trabalho de Ghosh e Nan$\mathrm{da}^{14}$ e Ngantung, Nanda e Bowman ${ }^{35}$, como observado na figura 3. Para controle de erro, dois traçados cefalométricos (traçados 1 e 2) de cada radiografia, pré- e pós-distalização com o aparelho Distal Jet, foram realizados pelo mesmo operador, com intervalo de duas semanas entre eles. $O$ teste estatístico de Wilcoxon foi empregado a fim de verificar a confiabilidade dos traçados 1 e 2, observando-se a ausência de diferença estatística entre os mesmos. Sendo assim, elegeu-se o traçado 1 para comparação das radiografias pré- e pós- distalização com o Distal Jet. 
As diferenças entre os traçados 1 pré- e pósdistalização foram novamente verificadas pelo teste estatístico de Wilcoxon, para avaliar os efeitos do aparelho Distal Jet sobre cada variável. Todos os resultados foram considerados significativos, para uma probabilidade de significância inferior a $5 \%(\mathrm{p}<0.05)$, tendo, portanto, pelo menos $95 \%$ de confiança nos resultados apresentados.

\section{RESULTADOS}

Segundo a tabela 1, pode ser observado que, de todas as variáveis, as únicas que apresentaram diferença estatisticamente significante $(\mathrm{p}<0.05)$ entre o pré e pós-distalização foram: SN - Segundo molar superior, PTV - Centróide do primeiro molar superior e PTV - Centróide do segundo molar superior. Isto sugere, que o aparelho Distal Jet foi capaz de distalizar tanto o primeiro quanto o segundo molar superior, inclinando para a distal apenas a coroa do segundo molar superior, sem uma perda significativa de ancoragem ou alterações no posicionamento espacial da mandíbula.

\section{DISCUSSÃO}

Antes de qualquer consideração e análise dos resultados deste estudo, deve-se enfatizar a dificuldade em se obter uma padronização absoluta, quando são utilizados seres humanos, como objeto de estudo. Especificamente na Ortodontia, por mais que se agreguem variáveis e medidas, sejam elas faciais, dentárias ou esqueléticas, com o objetivo de agrupar indivíduos semelhantes, não se pode mensurar parâmetros de incontestável valor no processo da correção ortodôntica, como por exemplo: a constituição bioquímica dos tecidos envolvidos e o potencial de crescimento. Sendo assim, todo trabalho que envolve amostras com seres humanos, traz intrínseco, o problema referente a uma adequada padronização.

Levando em consideração as possibilidades tecnológicas disponíveis atualmente, o presente estudo busca apresentar uma padronização superior aos demais estudos, disponíveis na literatura, principal- mente, no que tange as características esqueléticas. Ghosh e Nanda ${ }^{14}$, Runge, Martin e Bukai ${ }^{37}$, Gulati, Kharbanda e Parkash ${ }^{21}$, e Ngantung, Nanda e Bow$\operatorname{man}^{35}$ não relataram padronização das características esqueléticas sagitais e verticais das amostras, utilizadas em seus respectivos estudos.

Com relação à padronização da relação molar de Classe II dentária, os trabalhos que investigaram os efeitos dos distalizadores intrabucais ${ }^{8,14,21,35,37}$ e o presente estudo, não determinam a extensão desta Classe II. Teoricamente, uma relação completa de Classe II necessita de mais tempo para ser corrigida e, conseqüentemente, vai exigir mais da ancoragem, do que uma relação de topo-a-topo. Sendo assim, todos os estudos que se propõem a avaliar um mecanismo distalizador de molares superiores, deveriam padronizar a relação molar.

Uma deficiência específica do presente estudo, encontra-se no pequeno número da amostra, que trabalhou com 6 pacientes. Ainda que análises estatísticas próprias para amostras pequenas sejam utilizadas, com as devidas correções matemáticas, sabe-se da possibilidade de haver dados questionáveis, tendo em vista o peso estatístico, que os resultados com valores extremos representam para uma amostra pequena. No entanto, Bondemark e $\mathrm{Kurol}^{4}$, e Gulati, Kharbanda e Parkash ${ }^{21}$ avaliaram os magnetos repelentes e o Jones Jig, respectivamente, com uma amostra de 10 pacientes. Outros autores apresentaram resultados baseados em relatos de $\operatorname{casos}^{9,22,27}$.

Como o próprio nome explicita, este trabalho se constitui como um estudo piloto. A metodologia e os resultados encontrados, servem, em última análise, para elaboração de um estudo posterior, com uma amostra mais significativa e, sobretudo, mais padronizada. Procura-se, a seguir, interpretar os resultados obtidos, confrontando-os com os resultados encontrados por outros pesquisadores que utilizaram o aparelho Distal Jet, procurando, sobretudo, ponderar sobre os dados muito positivos, ou muito negativos. Além disso, são comparados os resultados deste aparelho, com os outros 
Tabela 1 - Análise descritiva e comparativa entre as medidas cefalométricas do pré- e pós-tratamento com o Distal Jet.

\section{Medidas}

Cefalométricas

Tecido Mole (mm)

Lábio Superior

para Plano E

Lábio Inferior

para Plano E

Esqueléticas

Ângulo SN-

Plano Palatino (graus) Ângulo SN-

Plano Oclusal (graus)

Ângulo Frankfurt

Plano Mandibular (graus)

PTV Ponto A

(mm)

PTV Ponto B

(mm)

ANS Mento

(mm)

Dentárias angulares (graus)

SN Incisivo

Superior

$\mathrm{SN} 1^{\circ}$ pré molar

superior

SN $1^{\circ}$ molar

superior

SN $2^{\circ}$ Molar

superior

Dentárias lineares (mm)

PTV Centróide

$1^{\circ}$ pré-molar

PTV Centróide

$1^{\circ}$ molar superior

PTV Centróide

$2^{\circ}$ molar superior

PTV Centróide

$1^{\circ}$ molar inferior

Plano palatino borda

incisal incisivo superior

Plano palatino Centróide

$1^{\circ}$ pré molar superior

Plano palatino Centróide

$$
1^{\circ} \text { molar superior }
$$

Plano palatino Centróide

$2^{\circ}$ molar superior

Plano mandibular Centróide

$$
1^{\circ} \text { molar inferior }
$$

$$
\text { Overjet }
$$

(Trespasse horizontal)

\begin{tabular}{|c|c|}
\hline Período & Míni \\
\hline Pré & $-1,5$ \\
\hline Pós & $-1,0$ \\
\hline Pré & 1,0 \\
\hline Pós & 2,0 \\
\hline Pré & 4,5 \\
\hline Pós & 4,0 \\
\hline Pré & 16 \\
\hline Pós & 15,0 \\
\hline Pré & 23, \\
\hline Pós & 23,0 \\
\hline Pré & 51, \\
\hline Pós & 50,5 \\
\hline Pré & 45,5 \\
\hline Pós & 44,5 \\
\hline Pré & 63, \\
\hline Pós & 63,0 \\
\hline Pré & 102, \\
\hline Pós & 104 \\
\hline Pré & 79,0 \\
\hline Pós & 77,0 \\
\hline Pré & 61, \\
\hline Pós & 58 \\
\hline Pré & 56,0 \\
\hline Pós & 52,5 \\
\hline Pré & 37,0 \\
\hline Pós & 40,0 \\
\hline Pré & 17,5 \\
\hline Pós & 14,0 \\
\hline Pré & 9,5 \\
\hline Pós & 6,5 \\
\hline Pré & 18 \\
\hline Pós & 18, \\
\hline Pré & 27,5 \\
\hline Pós & 28, \\
\hline Pré & 18,0 \\
\hline Pós & 18,5 \\
\hline Pré & 16,5 \\
\hline Pós & 16,0 \\
\hline Pré & 4,5 \\
\hline Pós & 6,0 \\
\hline Pré & 25,5 \\
\hline Pós & 26,0 \\
\hline Pré & 3,0 \\
\hline Pós & 4,0 \\
\hline Pré & 1,0 \\
\hline Pós & 0,5 \\
\hline
\end{tabular}

$$
\text { Overbite }
$$

(Trespasse vertical)

\section{Medidas Descritivas}

Máximo Mediana

\section{Média}

2,5

2,0

5,5

5,0

9,0

9,0

$$
20,0
$$

$$
19,0
$$

$$
34,5
$$

34,0

\section{4,5}

\section{4,5}

$$
60,5
$$

\section{0,5}

72,0

74,0

$-3,0$

0,5

2,0

2,5

\section{5,5}

6,5

$$
17,0
$$

\section{6,8}

\section{9,8}

28,8
52,3

\section{2,8}

\section{8,8}

\section{6,3}

\section{8,0}

70,8

\section{3,0}

\section{7,5}

\begin{tabular}{l|l}
111,5 & 108,5 \\
\hline
\end{tabular}

92,0

$$
94,0
$$

75,0

$$
75,0
$$

72,0

68,0

90,3

\section{7,5}

68,5

65,8
63,5

55,5

\begin{tabular}{|l|l|l|}
\hline 45,0 & 40,0 & 40,3 \\
\hline 47,5 & 41,0 & 41,8 \\
\hline
\end{tabular}

47,5

29,0

\section{7,0}

$$
\begin{aligned}
& 27,0 \\
& 17,0
\end{aligned}
$$

17,0
15,5

31,0

31,0

\section{2,0}

\section{2,5}

\section{5,5}

26,0

\begin{tabular}{|c|c|c|}
\hline 0,2 & 1,6 & 0,402 \\
\hline 0,6 & 1,1 & Pré $=$ Pós \\
\hline 2,6 & 1,7 & 0,273 \\
\hline 2,9 & 1,2 & Pré = Pós \\
\hline 6,3 & 1,8 & 0,423 \\
\hline 6,5 & 1,9 & Pré = Pós \\
\hline 17,7 & 1,5 & 0,201 \\
\hline 16,9 & 1,4 & Pré =Pós \\
\hline 29,3 & 4,1 & 0,059 \\
\hline 28,4 & 4,2 & Pré = Pós \\
\hline 52,7 & 1,3 & 0,893 \\
\hline 52,6 & 1,4 & Pré $=$ Pós \\
\hline 50,8 & 5,6 & 0,201 \\
\hline 49,6 & 6,5 & Pré $=$ Pós \\
\hline 68,0 & 3,5 & 0,059 \\
\hline 69,7 & 4,2 & Pré = Pós \\
\hline
\end{tabular}

\section{2,0}

22,5

\section{6,5}

16,5

28,0

27,5

6,5

9,0

6,0

4,5 d. $p$

107,2

108,3

88,2

87,5

68,3

66,1

64,2

57,9

40,3
41,8

23,0

19,9

13,5

11,3

23,5

22,8

30,2

30,4

22,0

23,4

18,6

18,8

9,8

11,1

26,8

26,6

5,4

6,5

3,9

2,9

Nota: 0 valor de $\mathrm{p}$ refere-se à probabilidade de significância do teste de Wilcoxon. 
mecanismos de distalização intra-arco, mais comumente citados na literatura.

Constatou-se que os primeiros e os segundos molares superiores apresentaram respectivamente 3,1 $\mathrm{mm}$ e 2,2 $\mathrm{mm}$ de distalização, num intervalo de tempo de 4,5 meses. Esses valores foram significativos, com $\mathrm{p}=0,036$. Ngantung, Nanda e Bowman $^{35}$ encontraram $2,12 \mathrm{~mm}$ e $2,58 \mathrm{~mm}$ para o primeiro e segundo molares respectivamente, num intervalo de tempo de 6.7 meses. Uma justificativa plausível, para explicar essa diferença na taxa de distalização entre o estudo empreendido e o desses autores, talvez seja a utilização do aparelho fixo superior, durante a fase ativa de distalização com o Distal Jet, no que tange o trabalho realizado por Ngantung, Nanda e Bowman ${ }^{35}$, o que pode ter aumentado a resistência de movimentação distal do molar pelo atrito entre o fio e o tubo do molar.

Comparando-se as taxas de distalização encontradas, com os outros mecanismos intra-bucais, observa-se valores similares àqueles reportados para o Pendulum ${ }^{14}$ e magnetos repelentes ${ }^{4}$; no entanto, eles foram maiores do que os resultados encontrados para o Jones $\mathrm{Jig}^{37}$.

Similarmente a Ngantung, Nanda e Bowman ${ }^{35}$, os resultados obtidos no presente estudo confirmam a superioridade do Distal Jet, sobre os outros aparelhos distalizadores intra-bucais, no que diz respeito ao pequeno componente de inclinação sobre o primeiro molar superior. Este dente apresentou uma inclinação distal de coroa de $2,2^{\circ}$, insignificante estatisticamente $(\mathrm{p}=0,10)$, o que caracteriza um movimento distal de translação do molar como afirmado por Carano e Testa ${ }^{9}$ e, indiscutivelmente, mais desejável do que os valores de $8,36^{\circ}$ e $14,5^{\circ}$ relatados, respectivamente, por Ghosh e Nanda ${ }^{14}$ e Byloff e Darendeliler ${ }^{8}$ para o

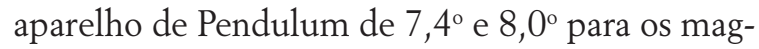
netos repelentes ${ }^{4,25}$ e $11^{\circ}$ para o Jones Jig ${ }^{13}$.

Já o segundo molar superior apresentou uma inclinação distal de coroa de 6,3 graus, com significância estatística $(p=0,036)$. Ngantung, Nanda e Bowman ${ }^{35}$, também, encontraram inclinação distal no segundo molar superior, maior do que no primeiro molar, porém com um valor maior do que o encontrado no estudo realizado: 11,79.

Movimentos verticais do primeiro molar superior não foram encontrados no corrente estudo, em plena sintonia com os achados de Ngantung, Nanda e Bowman ${ }^{35}$. Já para o aparelho Jones Jig, Jones e White ${ }^{27}$ afirmaram que não existe controle sobre a extrusão dos molares. Semelhantemente, Bondemark, Kürol e Bernhold ${ }^{5}$ encontraram extrusão do primeiro molar superior para os magnetos repelentes. Com relação ao Pêndulo há controvérsia. Ghosh e Nanda ${ }^{14}$ não encontraram alterações verticais no primeiro molar superior, enquanto Byloff e Darendeliler ${ }^{8}$ relataram intrusão de $1,68 \mathrm{~mm}$ deste mesmo dente.

A rotação axial e a distância inter-molar não foram avaliadas, neste estudo. $\mathrm{Na}$ realidade, essa se constitui como uma limitação particular, quando se utiliza apenas instrumentos de análise bidimensionais, como as radiografias cefalométricas utilizadas nesta pesquisa. Dessa forma, avaliações nos modelos de gesso devem ser contempladas, em estudos futuros, a fim de se avaliar a natureza do movimento molar no aspecto horizontal.

A perda de ancoragem nos sistemas distalizadores intra-arco está muito bem documentada, manifestando-se no aumento do movimento mesial (linear e angular) do pré-molar de ancoragem e no aumento do trespasse horizonta ${ }^{14,8,14,37}$. A ancoragem nesta modalidade de distalizadores é constituída, basicamente, de um apoio no palato, através do botão de Nance, com suas diversas variações, fixados aos segundos ou primeiros prémolares. Na realidade, esta ancoragem utilizada isoladamente não é capaz de resistir, completamente, à força mesial recíproca desses aparelhos distalizadores ${ }^{10,16,17,25}$. Os resultados de Ngantung, Nanda e Bowman ${ }^{35}$, previsivelmente, confirmaram a fragilidade da ancoragem intra-arco também para o aparelho Distal Jet.

No presente estudo, surpreendentemente, não foi constatado estatisticamente qualquer sinal de 
perda de ancoragem, visto que utilizamos os primeiros pré-molares como ancoragem com molas de 240 gramas. No entanto, os valores estão muito próximos do limite estatístico $(\mathrm{p}<0,05)$ para PTVcentróide do $1^{\circ}$ pré-molar superior $(\mathrm{p}=0,075)$, que mede o seu movimento mesial; e PP- centróide do $1^{\circ}$ pré-molar superior $(\mathrm{p}=0,059)$, que mede a extrusão do mesmo. Embora não se tenha observado perda de ancoragem com significância estatística, acredita-se que existe uma grande tendência de perda de ancoragem com o uso deste aparelho e, provavelmente, isto só não foi constatado, devido ao número reduzido da amostra $(\mathrm{n}=6)$. Vale ressaltar, que um dos nossos pacientes apresentou importante mesialização dos primeiros pré-molares e incisivos superiores, refletindo no aumento do trespasse horizontal anterior.

Isto sugere a necessidade de adicionar forças auxiliares, para restringir a perda de ancoragem anterior como, por exemplo, a utilização do aparelho extra-bucal ${ }^{10}$ e/ou elásticos de Classe II $^{15}$.

Observou-se clínica e cefalometricamente, ao final da distalização, que os segundos pré-molares, presentes na cavidade bucal ou ainda intra-ósseo, acompanharam o movimento do primeiro molar superior, como também observaram Freitas et al. ${ }^{13}$, Gianelly, Vaitas e Thomas ${ }^{17}$ e Everdi, Koyutürk e Küçükkeles ${ }^{12}$, quando utilizaram o primeiro prémolar como dente de ancoragem. Isto ocorreu, provavelmente, pela ação das fibras transeptais ${ }^{13}$.

O incisivo superior não apresentou alterações, estatisticamente significativas, assim como o lábio superior e inferior. Contrariando resultados encontrados neste estudo, Ngantung, Nanda e Bowman $^{35}$ encontraram um aumento de $12,16^{\circ}$ na inclinação vestibular do incisivo superior, e protrusão de $0,84 \mathrm{~mm}$ no lábio superior e 2,08 mm no lábio inferior. Esse resultado pode ser atribuído ao uso simultâneo de aparelho fixo total superior, pré-ajustado para alinhamento e nivelamento, durante o período de distalização, como os próprios autores relataram.

Assim como o reportado por Ngantung, Nan- da e Bowman ${ }^{35}$, este estudo não indicou qualquer efeito esquelético, com o uso do aparelho Distal Jet, seja na maxila ou na mandíbula. Resultado esse, similar aos dados referentes a outros dispositivos de distalização intra-arco $8,13,14,25,37$.

No trabalho conduzido por Ngantung, Nanda e Bowman ${ }^{35}$, a medida ANS - mento aumentou e o trespasse vertical sofreu diminuição, o que caracteriza um aumento na altura facial anterior inferior, efeito que, geralmente, é desfavorável para um caso de Classe II. Da mesma maneira, Ghosh e Nanda ${ }^{14}$ identificaram um aumento deste ângulo para o Pêndulo, e Runge, Martin e Bukai ${ }^{37}$ para o Jones Jig.

Neste estudo, apesar do aumento de 1,7 mm do ANS-mento não ser significativo estatisticamente $(p=0,059)$, encontra-se muito próximo do limite estatístico $(\mathrm{p}<0,05)$. De qualquer maneira, parece existir uma possível tendência de aumento da altura facial anterior inferior, resultante da tendência de extrusão de $1,4 \mathrm{~mm}(\mathrm{p}=0,059)$ presente no pré-molar de ancoragem.

Concordando com Ngantung, Nanda e Bow$\operatorname{man}^{35}$, o presente estudo também não registrou variação estatística do FMA. A título de curiosidade, surpreendentemente, este ângulo diminuiu $0,9 \mathrm{~mm}$ com um $\mathrm{p}=0,059$. Este valor está muito próximo do limite de significância, o que possivelmente indicaria uma tendência de diminuição do ângulo FMA. A única explicação plausível para esta possivel tendência, seria a ocorrência de um crescimento mais anterior do côndilo (provocando rotação anti-horária da mandíbula) manifestado, nesta amostra, durante o período de observação, visto que o AFAI aumentou, sem relação direta com um giro horário da mandíbula.

Similarmente, Runge, Martin e Bukai ${ }^{37}$ constataram, para o Jones Jig, um aumento do AFAI, não associado a um aumento do ângulo FMA. No entanto, esses autores ponderam sobre as limitações do estudo realizado, já que a amostra foi pequena $(\mathrm{n}=13)$. Ghosh e Nanda ${ }^{14}$, utilizando uma amostra bem mais expressiva $(n=31)$ para avaliar o Pendu- 
lum, constataram aumento, tanto do AFAI, quanto do ângulo FMA, configurando, claramente, uma rotação horária da mandíbula.

\section{CONCLUSÕES}

Os resultados deste estudo mostraram que o aparelho Distal Jet se constitui como um método eficiente na correção da relação molar de Classe II dentária, através da distalização de corpo dos molares superiores, ou seja, movimento distal de translação do primeiro molar superior.

Assim como os demais aparelhos distalizadores intra-arco que independem da cooperação do paciente, como o Pendulum, o Jones Jig e os magnetos repelentes, o Distal Jet parece compartilhar com a mesma insuficiente ancoragem. Assim sendo, a utilização de outros mecanismos para reforço de ancoragem, como elásticos ou aparelho extrabucal pode ser necessária, em alguns casos, nos quais a perda de ancoragem se tornar significativa durante a distalização.

É necessária a produção de mais estudos e pesquisas sobre o Distal Jet, utilizando, sobretudo, somente este aparelho, além, é claro, de amostras mais significativas e padronizadas, a fim de se traçar um retrato, mais fidedigno dos efeitos causados por esta mecânica e pela suas variações (primeiros ou segundos pré-molares como ancoragem, molas de $180 \mathrm{~g}$ ou $240 \mathrm{~g}$ e presença ou ausência de segundos molares superiores). Além disso, avaliações nos modelos de gesso devem ser contempladas a fim de se avaliar a natureza do movimento molar no aspecto horizontal, determinando então os seus efeitos nos três planos do espaço.

Enviado em: Dezembro de 2002 Revisado e aceito: Maio de 2003

\title{
Radiographic evaluation of the effects of Distal Jet appliance in intraoral distalizations: a pilot study
}

\begin{abstract}
The treatment of Class II malocclusion frequently requires maxillary molars' distalization. The extraoral appliance was the first created with this aim. It has proved its efficiency along the course of more than a century of its use. It presents, however, as a great disadvantage, the negative esthetic aspect, since its results depend on systematic patient cooperation. Thus, many intraoral distalizing appliances were developed with the specific aim of eliminating the need of patient cooperation. The one of them is the Distal Jet. According to Carano and Testa (1996) it is the only intraoral mechanism capable of distalizing molar of bodily. A pilot study was developed to evaluate the effect of this appliance. It used six adolescents (average age: 13.08), who had dental Class II malocclusion and a standard face pattern in common. Lateral headfilms were used before and after the treatment. The average time length was 4.5 months. Soft tissues, skeletal and dental changes were determined, comparing the measurements of the initial and final cephalograms. The items that varied significantly according to the Wilcoxon test were crown distal tipping of the second molar, distal movement of the first molar and distal movement of the second molar. The Distal Jet proved itself to be efficient in the upper molars' distalization mainly by the small component of distal tipping of the first molars. The anchorage loss and the increase in the lower anterior face height did not present statistical significance.
\end{abstract}

Key words: Class II malocclusion. Intraoral distalizing appliances. Distal Jet. 


\section{REFERÊNCIAS}

1. AAO issues special bulletin on extra oral appliance care, Editorial. Am J Orthod, St. Louis, v. 68, no. 4, p. 457, Oct. 1975.

2. ARMSTRONG, M. M. Controlling the magnitude, direction and duration of extraoral force. Am J Orthod, St. Louis, v. 59, no. 3, p. 217-243, Mar. 1971

3. BARTSCH, A.; WITT, E.; SAHM, G.; SCHNEIDER, S. Correlates of compliance. Am J Orthod Dentofacial Orthop, St. Louis, v. 104 , no. 4 , p. $378-386$, Oct. 1993

4. BONDEMARK, L.; KUROL, J. Distalization of maxillary first and second molars simultaneously with repelling magnets. Eur J Orthod, London, v. 14, no. 4, p. 264-272, 1992.

5. BONDEMARK, L.; KUROL, J.; BERNHOLD, M. Repelling magnets versus superelastic nickel-titanium coils in simultaneous distal movement of maxillary first and second molars. Angle Orthod, Appleton, v. 64, no. 3, p. 189-198, 1994.

6. BOWMAN, S. J. Correção de classe II e ortodontia para o paciente não cooperador. J Bras Ortodon Ortop Facial Curitiba, v. 3, no. 17, p. 23-35, 1998

7. BOWMAN, S. J. Modifications of the Distal Jet. J Clin Orthod Boulder, v. 32, no. 9, p. 549-556, Sept. 1998.

8. BYLOFF, F. K.; DARENDELILER, M. A. Distal molar movement using the pendulum appliance. Part 1: clinical and radiologica evaluation. Angle Orthod, Appleton, v. 67, no. 4, p. 249-260, 1997.

9. CARANO, A.; TESTA, M. The Distal jet for upper molar distalization. J Clin Orthod, Boulder, v. 30, no. 7, p. 374-380, July 1996.

10. CETLIN, N. M.; TEN HOEVE, A. Nonextration treatment. J Clin Orthod, Boulder, v. 17, no. 6, p. 396-413, June 1983.

11. CHAUSHU, G.; CHAUSHU, S.; WEINBERGER, T. Infraorbital abscess from orthodontic headgear. Am J Orthod Dentofacial Orthop, St. Louis, v. 112, no. 4, p. 364-366, Oct. 1997

12. EVERDI, N.; KOYUTÜRK, O; KÜÇÜKKELES, N. Nickel-titanium coil springs and repelling magnets: a comparison of two different intra-oral molar distalization techniques. Br J Orthod, London, v. 24, no. 1, p. 47-53, Feb. 1997.

13. FREITAS, B. V.; GARCIA, C. N. F.; GOLDENBERG, F. C. VIGORITO, M. S. M.; VIGORITO, J. W. Distalização unilateral de primeiros molares superiores com o aparelho Jones Jig. Apresentação de dois casos clínicos. Ortodontia, São Paulo v. 28, n. 3, p. 31-40, set./dez. 1995

14. GHOSH, J.: NANDA, R. S. Evaluation of na intraoral maxillary molar distalization technique. Am J Orthod Dentofacial Orthop, St. Louis, v. 110, no. 6, p. 639-646, Dec. 1996.

15. GIANELLY, A. A. Distal movement of the maxillary molars. Am J Orthod Dentofacial Orthop, St. Louis, v. 114, no. 1, p. 6672, July 1998.

16. GIANELLY, A. A.; BEDNAR, J.; DIETZ, V. S. Japanese NiTi coils used to move molars distally. Am J Orthod Dentofacial Orthop, St. Louis, v. 99, no. 6, p. 564-566, June 1991

17. GIANELLY, A. A.; VAITAS, A. S.; THOMAS, W. M. The use of magnets to move molars distally. Am J Orthod Dentofacial Orthop, St. Louis, v. 96, no. 2, p. 161-167, Aug. 1989.

18. GIANELLY, A. A.; VAITAS, A. S.; THOMAS, W. M.; BERGER, D. $G$. Case report: distalization of molars with repelling magnets. J Clin Orthod, Boulder, v. 22, no. 1, p. 40-44, Jan. 1988.

19. GOULD, E. Mechanical principles in extra oral anchorage. Am J Orthod, St. Louis, v. 43, no. 5, p. 319-333, May 1957

20. GREENSPAN, R. A. Reference charts for controlled extra- oral force to maxillary molars. Am J Orthod, St. Louis, v. 58, no. 5 p. 486-491, May 1970

21. GULATI, S.; KHARBANDA, O. P.; PARKASH, H. Dental and skeletal changes after intraoral molar distalization with sectional jig assembly. Am J Orthod Dentofacial Orthop, St. Louis, v. 114, no. 3, p. 319-327, Sept. 1998.

22. HILGERS, J. J. The pendulum appliance for class II non-compliance therapy. J Clin Orthod, Boulder, v. 26, no. 11, p. 706714, Nov. 1992

23. HENRY, R. G. A Classification of Class II, Division 1 Malocclusion Angle Orthod, Appleton, v. 27, no. 2, p. 83-92, Apr. 1957.
24. HOLLAND, G. N.; WALLACE, D. A.; MONDINO, B. J.; COLE, S. H.: RYAN, S. J. Severe Ocular Injuries from Orthodontic Headgear. J Clin Orthod, Boulder, v. 19, no. 11, p. 819-822, Nov. 1985.

25. ITOH, T.; TOKUDA, T.; KIYOSUE, S.; HIROSE, T.; MATSUMOTO, M.; CHACONAS, S. J. Molar distalization with repelling magnets. J Clin Orthod, Boulder, v. 25, no. 10, p. 611-617 Oct. 1991

26. JACOBSON, A. A key to the understanding of extra oral forces. Am J Orthod, St. Louis, v. 75, no. 4, p. 361-386, Apr. 1979.

27. JONES, R. D.; WHITE, J. M. Rapid class II molar correction with an open-coil jig. J Clin Orthod, Boulder, v. 26, no. 10, p. 661664, Oct. 1992

28. KLEIN, P. L. An Evaluation of Cervical Traction on the Maxilla an the upper First Permanent Molar. Angle Orthod, Appleton, v. 27 , no. 1, p. 61-68, Jan. 1957

29. KLOEHN, S. J. Evaluation of cervical anchorage force in treatment. Angle Orthod, Appleton, v. 31, no. 2, p. 91-104, Apr. 1961

30. KLOEHN, S. J. Orthodontics: force or persuasion. Angle Orthod, Appleton, v. 23, no. 1, p. 56-66, 1953.

31. LACERDA, C. R. V. Métodos para distalização dos molares superiores que utilizam mínima colaboração do paciente. 1999. 96 f. Trabalho de Conclusão de Curso (Especialização em Ortodontia e Ortopedia Facial)-Faculdade de Odontologia Universidade de Itaúna, Itaúna, 1999.

32. LOCATELLI, R.; BEDNAR, J.; DIETZ, V. S.; GIANELLY, A. A. Molar distalization with superelastic NiTi wire. J Clin Orthod Boulder, v. 26, no. 5, p. 277-279, May 1992.

33. MOYERS, R. E. Ortodontia. Trad. Aloysio Cariello. 4. ed. Rio de Janeiro: Guanabara Koogan, 1991.

34. NANDA, R. S.; KIERI, M. J. Prediction of cooperation in orthodontic treatment. Am J Orthod Dentofacial Orthop, St. Louis, v. 102 , no. 1, p. 15-21, July 1992.

35. NGANTUNG, V.; NANDA, R. S.; BOWMAN, S. J. Posttreatment evaluation of the distal jet appliance. Am J Orthod Dentofacial Orthop, St. Louis, v. 120, no. 2, p. 178-185, Aug. 2001

36. OOSTHUIZEN, L.; DIJKMAN, J. F.P.; EVANS, W. G. A Mechanical Appraisal of the Kloehn Extraoral Assembly. Angle Orthod Appleton, v. 43, no. 3, p. 221-232, July 1973

37. RUNGE, M. E.; MARTIN, J. T.; BUKAI, F. Analysis of rapid maxillary molar distal movement without patient cooperation. Am J Orthod Dentofacial Orthop, St. Louis, v. 115, no. 2, p.153-157, Feb. 1998.

38. SAMUELS, R. H. A. A review of orthodontic face-bow injuries and safety equipment. Am J Orthod Dentofacial Orthop, St. Louis, v. 110, no. 3, p. 269-272, Sept. 1996.

39. SILVA FILHO, O. G. Cefalometria radiográfica. Bauru: Hospital de Pesquisa e Reabilitação de Lesões Lábio-palatais da USP, 1984. 114 f. Mimeografado.

40. SILVA FILHO, O. G.; ARTUSO, E. S. R.; CAVASSAN, A. O. CAPELOZZA FILHO, L. Distalizador "Jones Jig": um método alternativo para a distalização de molares superiores. R Dental Press Ortodon Ortop Facial, Maringá, v. 5, n. 4, p. 18-26 jul./ago. 2000

41. TULLOCH, J. F. C.; PHILLIPS, C.; PROFFIT, W. R. The effects of early intervention on skeletal pattern in class II malocclusion: a randomized clinical trial. Am J Orthod Dentofacial Orthop, St. Louis, v. 111, no. 4, p. 391-400, Apr. 1997

\section{Endereço para correspondência}

Giordani Santos Silveira

Rua Cláudio Manoel, 602 - apto 701, Funcionários

CEP: 30140-100 - Belo Horizonte - MG, Brasil

giordanisilveira@hotmail.com 